\title{
Oral Mucosal Immune Suppression, Tolerance and Silencing: A Mini-Review
}

\author{
Michael AB Naafs* \\ Department of Endocrinology, Naafs International Health Consultancy, Europe
}

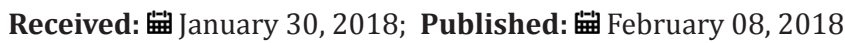

*Corresponding author: Michael AB Naafs, Deapartment of Endocrinology, Naafs International Health Consultancy, Europe

\section{Abstract}

The mucosal immune system spans the mucosal surfaces of the oro-pharyngeal cavity, gastrointestinal tract, respiratory tract, as well as the exocrine glands. In this mini-review the pathogenesis, pathophysiology of oral immunity, tolerance and resilence are discussed, with a special emphasis on the the oral microbiome in immune disorders.

Abbreviations: MALT: Mucosa-Associated Lymphoid Tissue; PPRs: Pattern Recognition Receptors; TLRs: Toll-Like Receptors; LPS: Lipopolysaccharides; PAMPs: Pathogen-Associated Molecular Patterns

\section{Introduction}

The oral-pharyngeal cavity is colonized by various microorganisms. In addition to microbes, food particles and external substances consumed through the oral cavity present potential challenges to the homeostasis of the oral mucosa. Hence, a mucosal membrane and inherent mucosal immune system are indispensable for the protection of the integrity of the internal environment. The mucosal immune system mounts immune response through immune cells residing in mucosal compartments. T lymphocytes residing in the mucosa play important roles in mucosal immunity and tolerance. In addition, $\mathrm{T}$ cell deficiency or defects in $\mathrm{T}$ cell function are associated with several oral mucosal diseases [1]. The mucosal immune system spans the mucosal surfaces of the oralpharyngeal cavity, gastrointestinal tract, respiratory tract, as well as the exocrine gands [1].

Despite differences in their location, the mucosal immune system at different organs share similar characteristics. Most basic knowledge comes from the better studied GI mucosal immune system [1,2]. The primary function of the immune system of the mouth is to protect the teeth, jaws, gingivae and the rest of the oral cavity against infection. The oral immune system is part of an extensive and specialized compartmentalized mucosaassociated lymphoid tissue (MALT)-[1]. The host defenses against infection vary in the different oral microenvironments or domains represented by the oral mucosa, salivary glands and saliva, and the gingival crevice. Despite high bacterial colonization and frequent allergen contact, acute inflammatory and allergic reactions are rarely seen in the oral mucosa. Therefore immune tolerance seems to predominate at this site. In this mini-review the pathogenesis, pathophysiology of oral immunity, tolerance and resilence will be discussed, with a special emphasis on the oral microbiome in (auto) mmune disorders.

\section{Pathogenesis}

The immune system is organized in tems of cells and molecukes that have specialized roles for defense against infection. Innate (natural) immunity and adaptive (acquired) immunity are two fundamental aspects of the immune response to eliminate microorganisms [3]. The innate immune system serves as a powerful barrier to invading bacterial pathogens by employing direct antimicrobial mechanisms, and indirectly, by stimulating the potent and antigen-specific adaptive immune response. The innate system is likely protective against many opportunistic pathogens and may increase the infectious dose of a successful pathogen required for symptomatic disease to occur [4]. Innate immunity lies behind most inflammatory responses. These are triggered in the first instance by macrophages, polymorphonuclear leukocytes, and mast cells through their innate immune receptors [5]. The components through the sensing structures ("pattern recognition receptors"-PPRs) initiate immunity receptors. An important 
class of these molecules are the Toll-like receptors (TLRs) which enable cells to recognize bacterial lipopolysaccharides (LPS) and other characteristic microbial molecules ("pathogen-associated molecular patterns"-PAMPs)-[6].

Adaptive immune responses are mediated by the generation of antigen-specific T and B cells. Antigens induce T cells in effector T cells that produce various cytokines or elicit cytolysis to eliminate target cells. On the other hand, B cells secrete immunoglobulins, which are responsible for eliminating extracellular microorganisms [3]. T lymphocytes are primarily responsible for cell-mediated immunity, and B lymphocytes are responsible for humoral immunity, but they work together and with other types of cells to mediate effective adaptive immunity. Along with the natural killer cells, these specialized lymphoid cells are derived from committed progenitors in hematopoietic tissues, which then undergo rearrangements of their antigen receptors to become clonically diverse lymphocytes. Newly formed $\mathrm{T}$ and $\mathrm{B}$ lymphocytes bearing autoreactive receptors can be eliminated by self-antigen contact in the thymus and bone marrow, respectively. The surviving $\mathrm{T}$ and B cells then migrate via the blood -stream to peripheral lymphoid tissues, where following antigen recognition, they may undergo clonal expansion and differentiation into effector $\mathrm{T}$ lymphocytes or antibody-producing plasma cells or otherwise become memory cells that await re-exposure to their specific antigens [7].

\section{Oral Mucosa}

The majoriy of pathogenic infectious agents enter the organisms by the muosal route. To cope with the enormous and highly variable antigenic load, resident cells are involved in uptake, processing and presentation of antigens, Production of antibodies and cellmediated defenses are strategically distributed at the front line of defense; the mucosal tissues and exocrine glands [6]. The parotid duct transports saliva from the gland into the oral cavity. The lamina propria of this gland presents granulocytes, $\mathrm{T}$ lymphocytes, and macrophages distributed in the lamina propria and epithelium and are encountered in the subepithelial cnnective tissue close to the oral cavity [8]. Moreover, the saliva contributes to the protection of the oral environment. Saliva contains several types of antimicrobial peptides and proteins (AMPs), including peroxidases, lactoferrin, lysozyme, histatins, phospholipase and calprotectin that mediate the innate response. Saliva contains also the AMPs defensins, (alpha and beta-defensins) [9,10]. The majority of immunologically active cells of oral tissue are the same present in the gastrintestinal (GI) tract [6]. Macrophages and dendritic cells located below the epithelium in the lamina propria are the first cells of the innate system to interact with microoorganisms and microbial products.

Macrophages are professional phagocytic cells that can internalize and kill bacteria by several mechanisms, some of which are part of the innate system such as phagocytosis, macropinocytosis and endocytosis [11], while others require the presence of specific antibodies against the bacterium and should be considered part of the effector arm of specific acquired immunity. Bacteria that are new to the host may activate the complement system by the alternative pathway, resulting in their opsonization and in a most efficient form of phagocytisis. Macrophages may also serve as antigen-presenting cells(APCs) in the initial steps of the induction of acquired immunity. They process the antigen and present it to the T-helper lymphocytes of an MHC2 (major histocompability complex) class molecule on the macrophages. Moreover, macrophages are considered a main source of the cytokines. interleukin (IL), IL-1 alpha, IL1-beta and TNF-alpha (tumor necrosis factor), which contribute to the initiation and regulation of the inflammatory process [12].

\section{Saliva And Salivary Glands}

The saliva of newborns is devoid of secretory IgA, however the concentration of secretory IgA rapidly increases and is close to that of adults by 1-2 years of age and certainly by 4-7 years [13]. Therefore, colonization with oral bacteria occurs in a mucosal environment that is immunologically responsive to infectious challenge. For example, by 3-5 weeks of age, salivary IgA responses specific for microorganisms that colonize the oral cavity at this time (such as S. mitis and S. salivarius ) can be detected [13]. By 12 months of age, both secretory IgA1 and secretory IgA2 specific for early colonizing streptococci are present.

\section{Gingival Crevice}

Even in healthy gingiva, there is a continous traffic of neutrophils from gingival capillaries into the gingival sulcus attracted by bacterial peptides from the biofilm of dental plaque and IL-8 from gingival epithelium [14]. Circulating blood leukocytes accumulate in the gingival tissues in response to dental plaque. The lymphocytes first become tethered to the endothelium of venules or other small blood vessels. This requires coupling of lymphocyte membrane receptor integrins binding to vascular endothelial ligands, , such as a variety of adhesion molecules. Once tethered, the lymphocytes then roll along the endothelial surface, attached by integrins $[15,16]$. Neutrophil polymorphs are induced to slow and then migrate through the blood vessel wall (diapedesis) In inflammation, histamine from mast cells or thrombin are released, resulting in increased expression of endothelial cell selectins (P selectin and later E selectin ), which pair with specific ligands on the neutrophil membrane. Endothelial cell "platelet activating factor"(PAF-1) is also up-regulated and binds to a specific neutrophil receptor.

Emigration of these activated neutrophils from the blood vessels is driven by $\mathrm{C} 5 \mathrm{a}$ (complement factor 5 ) fragments and leukotriene B4. Subsequently, the inflammatory reaction is continued by macrophages elaborating a spetrum of molecules including IL-1 and TNF acting on the endothelial cells which form selectins. In this later phase neutrophil emigration is also directed by IL- 8 and up-regulated by IL-1 and TNF. Most of the neutrophil polymorphs entering the gingival sulcus by these mechanisms are functionally active and capable of phagocytosis and the killing of microorganisms [17]. Conversely, quantitative neutrophil 
deficiencies, as in neutropenia result in uncontrolled apical extension of dental plaque and loss of periodontal attachment [18]

\section{Activation of the Oral Immune Response}

This starts with phagocytosis of antigens by macrophages and dendritic cells in lymphoid tissue or mucosal Langerhans cells. These cells process the antigens internally and present antigen peptide fragments assiocated with MHC- 2 molecules. The antigen presenting cells (APCs) briefly link up with any T cells they meet by means of intercellular adhesion molecules (ICAMS) 1 and 3 binding to the integrin LFA-1 (leukocyte functioning antigen) Most $\mathrm{T}$ cells have surface receptors made up of a heterodimer alpha and beta- chain with highly variable regions, in an immunoglobulin-like configuration, conferring antigen specificity. A minority of T cells have gamma and delta chain receptors instead and this subset is relatively more numerous in sites such as the tongue. Recognition of antigen associated with the MHC-2 class molecules on the APC by the $\mathrm{T}$ cell receptor provides a first signal, but for full activation of resting helper cells a second signal is needed [19].

In the first signal the APCs migrate to lymph nodes, where they present antigenic peptides to naive (never exposed to antigen), yet epitope-specific CD4+ T lymphocytes (T helper cells). CD4+ lymphocyte activation requires the interaction of specific $\mathrm{T}$ cell receptors with antigen peptide-MHC-2 class complexes on the APCs. The second signal is formed by the APCs and the ligation of co-stimulating receptors of the CD 28 family on T cells by B7 family members of co-stimulating molecules (CD80 and CD86) on the APC, a ligand for CD28 on the T cell and IL-1 from the APC [20]. They are needed for full activation of the resting T helper cells [19]. The helper T lymphocyte response is MHC restricted and CD4 surface molecules on the Thelper cells associate with MHC-2 molecules on the macrophages. T cell CD2 is always involved in this T cell-APC interaction. The switched on T-cells now synthesize IL-2, which has an autocrine effect via receptors in triggering $\mathrm{T}$ cell proliferation. Other $\mathrm{T}$ cell cytokines released include macrophage-colony stimulating factor (GM-CSF), IL-4 and TNF-beta.

In addition to presenting antigens dendritic cells (DCs), can polarize naive T cells ( $\mathrm{T} 0$ cells) in either Th1 or Th2 cells, according to their own phenotype, and with signals from processed antigens and from the tissue environment during antigen presentation. Contacts with a virus promote a Th1 phenotype and cytokines as IL-3, prostaglandines E2 and thymic stromal lymphopoeitin released from epithelial cells promote a Th2 phenotype [21]. A distinct subtype of $\mathrm{T}$ cells, the so called regulatory $\mathrm{T}$ cells ( Tregs) suppress immune response (both Th2 and Th1) through the inhibitory cytokines and cell surface molecules, including IL10 and transforming growth factor-beta. (TGF-beta). cytotoxic T lymphocyte antigen-4 (CTLA-4) and programmed death-1 (PD-1). Tregs can also inhibit effector $\mathrm{T}$ cells via a direct cell-cell contact mechanism to induce apoptosis. In addition, Tregs crosstalk with APCs to suppress T cell activation [22]. Tregs are categorized as natural or adaptive (inducible $\operatorname{Tr} 1$ ) The former are characterized by the expression of high levels of CD25 on their surface and by the transcription factor forkhead box P3 (FOXP3) [23]. Both nonallergic and allergic individuals retain allergic-specific IL-4 producing effector T cells, IL-10 producing TR1 cells and CD25 Tregs, but in different proprtions. Thus the balance between TH2 and certain Treg populations may decide whether clinical allergy will develop.

\section{Pathophysiology}

\section{Immunosuppression and the oral cavity}

Immunosupression is the deliberate prevention or reduction of an immune response. It can result from the administration of immunosupressive agents or from the deliberate depletion of immune cells, as well as from malnutrition, cancers and certain chronic infections, such as HIV (human immunodefieciency virus). An unwanted side effect is immunodeficiency.

\section{Transplantations}

Solid-organ transplantation in patients with end-stage organ failure requires lifelong immunosuppression. to prevent transplant rejection. The goal of immunosupressive therapy is to inhibit T-cell mediated responses, since CD4+ and, or CD8+ lymphocytes have a requisite role in graft rejection [24]. However opportunistic infections, which could directly impact graft survival, are a common comorbidity of continued immunosuppreesion [25,26]. Common causes of infection, particularily in the late post-transplantation period, are gram-negative rods such as Escherichia coli, Kliebsiella pneumoniae, and Pseudomonas aeruginosa, in addition to grampositive cocci such as Enterococcus spp. and Staphylococci $[26,27]$. Fungal infections, commonly caused by Candida spp. , are also frequent in this patient population [26]. While the source of infectious microorganisms could be exogenous, the endogenous flora, i.e., the microbiome, may also serve as an important reservoir of opportunistic infections.

Moreover, disrupted homeostasis of the oral microbiome could promote colonization by non-resident microorganisms or increase carriage of opportunistic bacteria and fungi, augmenting the possibility of their translocation to distal sites. Thus mucosal surfaces have the potential to become important infection portals or reservoirs. The oral cavity, in particular, harbors a diverse microbiome and represents a portal of entry for microorganisms in the host. The relationship between long-term, low -intensity immunosuppression aimed at limitting adaptive immune response and the residents colonizing mucosal surfaces is unclear. Studies that characterized the microflora at mucosal surfaces in chronically immunosurprised patients are scarce, with the available studies having used microbiological studies of limited scope [28,29]. The advent of rRNA gene-based taxonomic identification combined with-high-throughput sequencing technologies permits comprehensive characterization of the host microflora, providing a view of microbiome diversity not previously possible. High- 
throughput sequencing libraries do evaluate the effect of long-term immunosupression on the microbiome and are in use only since a few years [30].

Diazetal.found that pharmacologicalimmunosuppressionaimed at increasing allograft tolerance does not affect the most common and abundant bacterial species in saliva but increase the frequency of detection of microorganism known to be the cause of extra-oral infections in transplant recipients $[27,31,32]$. In their study $(n=39$; 20 transplant recipients and 19 non-immunosuppressed controls ) potentially opportunistic Gammaproteo bacteria, such as Klebsiella pneumoniae, Pseudomonas fluorescens, Acinetobacter species and the genera Acinetobacter and Klebsiella were more prevalent in the transplant recipients. Furthermore, in transplant subjects, the dose of the immunosuppressant prednisone correlated positively with bacterial richness [30].

\section{Autoimmune disease (AD)}

\section{Rheumatoid arthritis (RA)}

Rheumatoid arthritis (RA) is an inflammatory autoimmune disease strongly associated with periodontal disease [33]. In a study by Zhang et al. dysbiosis was evident in the gut and oral microbiomes of RA patients and there was a concordance between the two microbiomes. Differences in the gut and, or oral microbiomes distinguished individuals with RA from healthy controls and were used to stratify individuals according to their response to therapy [33]. The altered composition of the gut or oral microbiomes in individuals with RA correlated with the prognosis and diagnosis of the disease [33]. Moreover, in two separate and unrelated double-blind randomized placebo-controlled trials aimed to evaluate the benefit of oral probiotic (specific microbes thought to be benificial to the host) supplements as an adjunct microbial therapeutic intervention, the RA patients who recieved daily capsules of the probiotic Lactobacillus casei 01 over a 2 month period had statistically significant improvements in their disease activity and inflammatory status compared with controls [34-36].

\section{Primary Sjögren's Syndrome ( pSS)}

Dysbiosis has been hypothesized to play a role in the pathogenesis of autoimmune disease. Primary Sjögren's syndrome is an autoimmune disease characterized by sicca symptoms resulting from saliva and lacrimal dysfunction. This could result in dysbiosis of the oral cavity. At the same time dysbiosis could also be hypothesized to have a causative role. In a small study Sandhya et al. compared the oral microbiome of 13 pSS patients and 12 controls. Organisms significantly enriched in pSS included the following; (FC and p values); Capnocytophage (2, 09;0, 01) Dialister $(2,13 ; 0,02)$, Fusobacterium $(2,84,0,04)$, Helicobacter $(4$ 83, 003), Streptococcus $(3,33 ; 0,01)$, and Veilonella $(3,82,0,006)$ spp. A paucity of Pseudomonas was noted compared to controls. In addition, a distint subset of organisms was enriched in the oral cavity of patients with pSS. This subset includes Capnacytophaga, previously shown to be associated with the pathogenesis and $\mathrm{T}$ cell activation in pSS [37].

In another study, van der Meulen et al assessed whether changes in the oral microbiome of pSS patients are a cause of of pSS or an effect of oral dryness. They collected swabs from the buccal mucosa of 82 patients with oral dryness; 32 patients could be classified as pSS according to the 2002 AECG classification. Fiftyfive patients were classified as non-SS sicca. The control group consisted of a population based cohort study $(n=118)$. The bacterial composition of the buccal mucosal samples was determined with 16S rRNA sequencing. Multivariate analysis showed highly significant associations between oral dryness and the genera Olsenella, Cryptobacterium and Fretibacterium, all which have been associated with periodontal disease, since these genera were only detected in samples from the buccal mucosa of patients with oral dryness and not in population controls $(\mathrm{p}<0,05)$. However, no oral bacterial taxa were specifically associated with pSS [38].

\section{SLE}

Systemic Lupus Erythematosus (SLE) is associated with differences in the composition of the oral microbiota, independently of periodontal status [39]. Correa et al. evaluated 52 subjects with SLE compared to 52 subjects without SLE (control group). Subjects were classified as without and with periodontitis. Oral microbiota composition was assessed by $16 \mathrm{~S}$ rRNA gene sequencing from subgingival DNA dental plaque. SLE patients exhibited higher prevalence of periodontitis which occured at a younger age compared to subjects of the control group. More severe forms of periodontitis were found in SLE subjects that had higher bacterial loads and decreased microbial diversity. Bacterial species frequently detected in periodontal disease were observed in higher proportions in SLE patients, such as Fretibacterium, Prevotella nigrescens and Selomonas. Changes in the oral microbiota were linked to increased local inflammation, as demonstrated by higher concentrations of IL-6, Il-17 and IL-33 in SLE patients with periodontitis [39].

\section{Systemic sclerosis (SSc)}

The hallmark of SSc or scleroderma is a pathological fibrosis of skin and internal organs, The presence of autoantibodies supports the autoimmunity aspect of this disease and are utilzed in categorizing SSc in diffuse and limied forms. In a combined UCLA/ Olso University study the gut microbiome of 17 adults with SSc from UCLA, 17 from Oslo and of 17 controls was investigated. The people with systemic sclerosis had significantly lower levels of gut bacteria believed to protect against inflammation, such as Bacteroides (UCLA and Oslo), Faecalibacterium (UCLA) and Clostridiun (Oslo). They also had significantly higher levels of bacteria that promote inflammation, such as Fusobacterium (UCLA), compared with those 
in the control group. Increased levels of Clostridium was associated with less severe gastrointestinal disease [40]. Oral microbiome data of SSc patients are not yet available.

\section{Behcet's syndrome (BS)}

Behcet's syndrome (BS) is a multisystem immune-related disease of unknown etiology. Recurrent aphtous stomatitis (RAS) is characterized by the presence of idiopathic oral ulceration without extra-oral manifestations. The interplay between the oral microbial communities and the immune response could play an important role in the etiology of both BS and RAS. Seoidi et al investtigated the salivary and mucsal microbal communities in BS and RAS. Purified microbial DNA isolated from saliva samples (54 BS, 25 healthy controls(HC) and 8 RAS) were examined by the human oral microbe identification microarray. There was increased colonization with Rothia deuticariosa of the non-ulcer sites of BS and RAS patients $(\mathrm{p}<0,05)$ Ulcer sites in BS were highly colonized with Streptococcus salivarius compared to those of RAS $(\mathrm{p}<0.05)$ and with Streptococcus sanguis compared to $\mathrm{HC}(\mathrm{p}<0,001)$. Oral mucosa of HC were more highly colonized with Neisseria and Veillonella compared to all studied groips $(p<0,001)$. Despite the uncertainty whether the reported differences in the oral mucosal microbial community of BS and RAS are of causitive or reactive nature, it is envisaged that restoring the balance of the oral microbiota of the ulcer sites may be used in the future as a new treatment modality for oral ulceration [41].

\section{Ankylosing spondylitis (AS)}

Ankylosing spondylitis or Bechterew's disease is a chronic inflammatory disease primarily affecting the sacroilliac joints and the spine, for which the pathogenesis is thought to be a result of the combination of host genetic factors and environmental triggers. There are 100 trillion bacteria residing in the human gut having established a synbiotic relation with their host, influencing many aspects of host metabolism, physiology and immunity. A growing body of evidence suggests that intestinal microbiota may play an important role in AS [42]. In animal studies HLA-B 27 (human leukocyte antigen B 27) transgenic rats do not develop many features of SpAs (spondylarthropathies), when raised in a germ-free environment [43]. However, arthritis develops when commensal bacteria such as Bacteroides vulgaris, are introduced in these germfree models [44]. After transfer to a conventional rat colony HLA-B 27 transgenic rats appear with symptoms similar to AS.

Oral microbiota (with over 700 species being harbored in the oral cavity) have a role in periodontal disease and in turn, play a contribitory role in periodontitis [44]. Comparing AS patients with healthy people, AS patients have the higher level of antiPophyromonas gingivalis, suggesting potential interaction between some specific microbiota and AS [45-48]. Martinez et al. suggested infection was closely related with patients with SpA. They showed that $56 \%$ of patients had infections, including respiratory infections, urinary system infections, and intestinal infections. Moreover, HLA-B 27 positive patients have a higher infection rate, and the intestines are the most commonly involved [49]. About $70 \%$ of patients with AS have subclinical gut inflammation, which indicates that the two diseases may be similar entities with a common origin, that is gut dysbiosis [50-52].

Recently, it has been shown that the occurence of spondylarthritis is influenced by the intestinal microbiota [50-52]. The role of gut microbiome in AS pathogenesis is suggested by many studies $[50,53,54]$. AS can be associated with inflammatory bowel disease (IBD)-[53,54]. During the active stage of the disease, most of the patients exhibited an elevated serum total IgA level, suggesting microbial translation and intestinal barrier failure [50]. A recent study has indicated that in the terminal ileum, compared to healthy controls, AS patients had a higher abundance of 5 bacterial families (e.g., Lachnospiraceae, Prvotellaceae, Rikemellaceae, Porphyromonadoceae and Bacteroidaceae) and a lower abundance of two bacterial families (Ruminococcoceae and Rikemellaceae) [50]. An interesting report by Tito et al suggested that the status of intestinal inflammation associated strongly with the profile of mucosal microbiota of SpA patients and that Diallister could be a potential microbial biomarker of SpA disease activity [55].

\section{Vasculitides and Connective Tissue Disorders (CTDs)}

Numerous studies suggest that alterations in human microbiota may be involved in the pathogenesis of inflammatory arthritides as a result of the aberrant activation of the innate and adaptive immune response. Only a few studies have explored the involvement of dysbiosis in other CTDs or vasculitides [56]. No data are currently available concerning the microbial composition or pathogenic role of the microbiome in autoimmune myositides. There are no available data concerning the role of the microbiome in undifferentiated CTDs (UCTDs), mixed CTD (MCTD), relapsing polychondritis or other overlap syndromes. The vasculitides are a wide spectrum of disease characterized by chronic inflammation of small, medium-sized, , and large vessels. Healthy vessels may harbour their own commensal microbiome, but colonization of the vascular tree by pathogenic microbial agents, including Chlamydia pneumoniae, Porphyromonas gingivalis, Aggregatibacter actinomycetum comitans, Staphylococcus and Stenotrophomones spp. , has recently been described in a number of vascular diseases, including atherosclerosis and aneurysms [57]. So, vascular dysbiosis might contribute to the chronic inflammation occuring in systemic vasculitides.

Giant cell arteriitis (GCA) is a systemic granulomatous vasculitis affecting large vessels that mainly occurs in the elderly and has a typical cyclic pattern every 5-7 years. It has been postulated that an infectious stimulus may underlie the onset, but the etiological agent is still unknown. In a DNA sequencing study of the temporal 
artery biopsies of 17 GCA patients and 5 controls Bhatt et al. did not find evidence for any distinctive microbiome signature in the patients and the isolation of Propionibacterium acnes, Escherichia coli, and Moraxella cattarhalis in both GCA and healthy subjects samples mainly reflected contamination [58]. Kinumaki et al. [59] made a longitudinal metagnomic analysis to assess intestinal microbial variations in a cohort of 28 patients with Kawasaki disease (KD)-[59]. The authors found that 5 streptococcal species ( $\mathrm{S}$, pneumonia, pseudopneumonia oralis, gordonii, and sanguis), and in severe cases the genera Rothia and Staphylocoocus were the most abundantly represented mictoorganisms in the intestinal tract of KD patients, and their prevalence increased during the phases of disease reactivation. On the contrary, the presence of Ruminococcus, Blautria, Faecalibacterium and Roseburia bacteria increased during disease remission [59]. There are no published data concerning dysbiosis in small vessel vasculitides.

\section{Diabetes Mellitus}

There is growing evidence suggesting a two-way relationship between diabetes and oral health. Multiple large populationbased studies have reported that periodontal diseases were independently associated with an increased risk of diabetes $[60,61]$. Treatment of oral diseases have been shown to be associated with improved glycemic control and a reduction in $\mathrm{HbA1C}$ (glycosylated hemoglobin)-[62,63]. On the other hand, individuals with diabetes are more likely to have periodontitis with increased severity, when their diabetes is poorly controlled [64]. It has been suggested that systemic inflammation may be the link between pathogenesis and oral diseases $[65,66]$. There is also growing evidence suggesting that the oral microbiome plays an important role in obesity and diabetes. Studies have suggested a direct link between periodontal pathogenic bacteria (such as Porphyromonos gingivalis and Aggregati mycetocomitans) and glycemic control and diabetes risk [67-70]. However direct investigations of the relationship between the oral microbiome and diabetes have been limited. In a study of 29 morbidly obese subjects, including 13 patients with diabetes, the genus Bifidobacteria in the phylum Actinobacteria was shown to have a lower abundance in patients with diabetes [71]. In another study, including 20 diabetic cases and 11 controls, two genera, Streptococci and Lactobacilli, in the phylum Firmicates were found to $\mathrm{b}$ e more abundant in patients with diabetes [72].

These studies were limited by their small sample size and, or a limited number of bacteria species investigated, Long et al. studied the oral microbiome profiles of 98 participants with incident diabetes, 99 obese non-diabetics and 97 normal weight non-diabetics, via deep sequencing of the 16S rRNA gene [73]. They found that the phylum Actinobacteria was present significantly less abundant among patients with diabetes than among the controls $(\mathrm{p}=0,0039: \mathrm{OR}: \mathrm{CI} ; 0,27(0,11-0,66)$ for these individuals, who had relative abundance higher than the median value. Within this phylum, 5 familie and 7 genera were observed, and most of them were less abundant among patients with diabetes [73]. Notably, genera Actinomyces and Atopobium were associated with $66 \%$ and $72 \%$ decreased risk of diabetes ( $p<0,009$ and $p<0,007$, respectively) So, multiple bacteria taxa in the phylum Actinobacteria are associated with the risk of type 2 diabetes). Some are also associated with the prevalence of obesity, suggesting the oral microbiome may play an important role in diabetes etiology [73]. Disruption of intestinal microbiota transmission from mother to infant has been associated with type1 diabetes [74-77]. Oral microbiota data for type1 diabetes are not available, yet.

\section{Thyroid Disorders}

Autoimmune thyroiditis has an increased prevalence in patients with celiac disease and vice versa [78]. Hashimoto's thyroiditis (HT) is one of the most common autoimmune endocrine diseases. Celiac disease is associated with a number of autoimmune conditions, including HT. Changes in microbiota and short-chain fatty acuds (SCFAs) are clearly related to the pathogenisis of CD, but their role in thyroid immunity induction or protection remains to be investigated [79-87].

\section{HIV}

Mucosal surfaces account for the vast majority of HIV transmission. In adults, HIV transmission occurs mainly by vaginal and rectal routes, but rarely via the oral route. HIV infection could be the result of oral route by breastfeeding in pediatric HIV infections. HIV selectively infects, depletes and dysregulates multiple arms of the immune system, particularily at the mucosal sites. HIV infection is also associated with the prevalence of oral mucosal infection and dysregulation of the oral microbiota, both of which may compromise the oral mucosal immunity of HIVinfected individuals. In addition, impaired oral immunity in HIV infection may predispose the patients to periodontal diseases that are associated with systemic inflammation and increased risk of cardiovascular diseases. The persistence of immune activation and a pro-inflammatory environment is in part due to the leakiness of the gastrointestinal (GI) tract $[88,89]$. The GI tract is an important site of HIV pathogenesis and early in the disease, associated with massive depletion of $\mathrm{CD} 4+\mathrm{T}$ cells, causing irrepairable destruction of the epithelial lining of the GI tract (Among CD4+ T cells, T helper cells TH17 (Th17) cells mediate immunity against pathogens at mucosal surfaces, their depletion from the gut of HIV-infected individuals increases intestinal permeability and leads to microbial translocation [90-92]. Microbial translocation in HIV-Infected patients has been linked to preferential depletion of lymphocytes capable of producing the effector cytokines IL-17 and IL-22 $[88,93,94]$. It is unclear as to why the gut mucosa fails to recover even after the start of ART (antiretroviral treatment).

Since the gut-associated lymphoid tissue ( GALT) is so similar to the lymphoid tissue of the oral cavity, it would be hypothesized that HIV infection may result in similar patterns of distinction in 
the oral mucosa [95]. Oral mucosal immune cells express a wide range of pathogen-receptor=recognition receptors (PPRs) and metabolic sensors that act either as suppressors or activators [96]. The prevalence of periodontal disease may result in serious oral inflammatory conditions and paricularily for immunucompromised individuals $[97,98]$. It has been shown that people living with HIV are more likely than their HIV-negative counterparts to have serious oral health issues such as oral candidiasis, hairy leukoplakia, warts, aphthous ulcers and herpes even in the presence of ART $[99,100]$.

HIV infection appears to directly or indirectly impact the systemic and local immunity leading to oral opportunistic infections and malignancies [101]. For instance, higher expression of oral cytokeratin in HIV-infected individuals and patients on ART has been reported, which indicates transformation of the oral mucosa and increased risk of malignancy [102]. The role of oral microbiota in HIV-infected individuals requires further attention as either the disease (HIV) or the treatment (ART) may impact diversity and composition of the oral microbiome. For instance, it has been shown that elevated viremia in untreated patients is associated with significantly higher proportions of potentially pathogenic Veillonella, Prevotella, Megasphaera, and Campylobacter species [103]. Increased prevalence of these potential pathogens result in the diminished presence of commensal Streptococcus and Veillonella species [103].

However, ART treated patients showed lower colonization by Neisseria fluvescens compared with healthy controls [103]. Another study reported that microbial diversity in the oral cavity of HIV-infected individuals was lower than in healthy controls and this diversity was further reduced during ART [98]. By contrast, while the bacterial community of oral wash specimen in HIVinfected compared to controls, using a deep sequencing approach a difference in fungal communities was observed [104]. A recent study found that Haemophilus parainfluenza, which has been implicated in opportunistic infections, was associated with HIVinfected individuals [105]. A most recent study utilizing microarray and pyrosequencing techniques reported a significant difference in the prevalence and distribution of the saliva bacterial communities among HIV-infected individuals before and after initiation of ART [106]. This study found that Actinomyces, Atopobium and Aggregati bacteria genera were significantly different from the baseline after ART [106]. These evidences suggest that there is a shift in the oral microbiome and these changes might be associated with HIV infection and, or HIV treatment and other oral manifestations associated with disease.

One of the main limitations of these studies seems the limited number of studied subjects. Another problem is the lack of standardization in the landscape of microbiome techniques. There is a sense of urgency to optimize methods and to improve reproducibility between laboratories [107-109]. A large body of evidence has shown that common oral infections may play important roles in atherosclerosis [110] and an increasingly common chronic inflammatory state that is present in $10-15 \%$ of the world's population is periodontitis [111]. Clinical signs include deepening of the periodontal pocket surrounding the tooth, loss of attachment including the gingival mucosa, which will progressively lead to loosening of the teeth and ultimately tooth loss. The etiology of periodontitis is generally caused by local factors, such as accumulation of dental biofilm from poor oral hygiene practices, but can also be a manifestation of severe systemic disease. Individuals with HIV are more susceptible to developing periodontitis because of their compromised immune system.

Acute necrotizing periodontitis can also be a manifestation of a newly infected individual with HIV [112]. In fact, oral manifestations may be present in up to $50 \%$ of HIV-infected individuals, and up to $80 \%$ of those who have progressed to AIDS [112,113]. Looking at periodontitis from a molecular perspective it appears that the host's immune system in response to pathogens secretes substantial amounts of pro-inflammatory cytokines like IL-1beta, TNF-alpha, and IL-6 along with tissue destuctive mediators like oxygen intermediates and metalloproteinases [114]. The oral mucosal epithelial lining begins to ulcerate and forms an easy port of entry for microorganisms or their by-products in the blood stream [115]. Many of these bacteria are Gram-negative, obligate anaerobes and can colonize at distant sites [116]. Haraszthy et al tested 50 samples from patients undergoing carotid endarterectomy to determine if any periodontal bacterial pathogens were present. Forty four percent of the specimen were positive for at least one pathogen [116]. This suggests that oral microorganisms can enter the blood stream and induce the development and progression of systemic inflammation that may lead to other complications such as cardiovascular disease (CVD).

\section{Immune Tolerance}

Antigen exposure to oral mucosa is generally thought to lead to immune tolerance induction. However, very little is known about the subset composition and function of the dendritic cells (DC) migrating from human oral mucosa. As mentioned before, they are powerful antigen presenting cells (APCs) linking innate to adaptive immunity. They perform a delicate balancing act, maintaining immune tolerance under steady-state conditions, but also inducing $\mathrm{T}$ cell immunity when needed. During homeostasis migrating immature DCs from peripheral tissues take up antigen but do not acquire the capacity to promote functional T cell-mediated immune responses [117,118]. It is well established that inflammatory responses and allergic reactions can occur in the oral cavity and in the skin $[119,120]$. Extensive literature on mucosal tolerisation generally refers to the gut, which has a clear immunosupressive character compared to the inflammatory properties of the skin. When refering to "oral tolerance"often "gut tolerance"is actually meant [121-125]. 
Kosten et al:their skin counterparts. recently assessed the distribution, maturation state and functionality of human oral mucosa associated migratory DC subsets in a comparative analysis with thei r skin counterparts. Flowcytometric and $\mathrm{T}$ cell priming analysis showed a similar subset distribution and activation state among gingiva migrated DC, but surprisingly also revealed their superior type $-1 \mathrm{~T}$ cell skewing capacity. These data call for a reappraisal of the functionality of oral mucosa associated DC subsets and shed new light on oral tolerance and immunisation[126]. Cytokine release profiling pointed to a more pro-inflammatory microenvironment in the gingiva than in the skin.

The skin is commonly regarded as an attractive gateway to the delivery of (tumor) vaccines, whereas the oral mucosa is regarded as a gateway for the delivery of immuno-modulatory desentisation therapies e. g., subcutaneous immuno therapy (SCIT) and sublingual immunotherapy (SLIT) -[127]. So, it seems that rather than mediating immune tolerance, like the gut, the oral mucosa has immune stimulatory properties akin and superior to the skin [126]. One could argue that like skin the mouth is a gatekeeper and major barrier to the outside world where strong immune defences should be up all times, while the gut may be more important in preventing chronic inflammation. More studies to support this hypothesis are warranted, however [126].

\section{Immune Resilence}

Most of research concerning the influence of microbiota on immune function has been performed in gut microbiota [76]. Gut microbiota play a role in modulating the immune response both locally and systemically [128]. In the absence of commensal bacteria GF (germ-free) mice have impaired development of the innate and adaptive immune system $[129,130]$ reduced number of IgA producing plasma cells and a decreased percentage of CD4+ T cells [131]. Additionally, T helper Th17 cells, which produce pro- inflammatory cytokines are regulated by gut bacteria and are promoted specifically by segmented filamentous bacteria (SFB)-[132]. Autoimmune disease (AD) has been correlated with alterations of the microbiome (dysbiosis), as mentioned before. A different example linking the microbiota and immunity comes from a study in mice, which showed that Lactobacillus reuteri enhances wound-healing properties in the host through up- regulation of the neuropeptide hormone oxytocin by a vagus nerve-mediated pathway [133].

Stress by the central nervous system leads to release of a variety of hormones, neurochemicals and neuropeptides, which can directly or indirectly affect immune function, usually resulting in impairment [134]. Nearly all immune classes possess receptors for the stress-related neurohormones adrenaline and noradrenaline [135]. The ability of bacterial pathogens to influence behavior has been recognized for decades, most notably bacteria that invade the nervous system. The concept of the "gut microbiome-brain axis" developed in the early 1990's [135]. This gut microbiome-brain axis influences behaviors as emotions, anxiety, depression, appetite and metabolism, sepsis, besides immune function [76]. Although there are many similarities between the gut microbiome and the oral microbiome, data regarding a possible mouth microbiomebrain axis are not available, yet [136].

\section{Conclusion}

Our perception of the microbiome has changed rapidly the last decade due to the metagnomic sequencing of the DNA and RNA repertoire present in the intestinal ecosystem and the re-emergence of gnotobiotic approaches. Dysbiosis, both of the oral cavity and intestinal tract plays a role in periodontitis, autoimmune disorders, HIV infection, transplant allograft rejection, atherosclerosis, cardiovascular disease, diabetes mellitus and obesity While gastrointestinal dysbiosis is involved in immune resilence, behavior, appetite and metabolism, and sepsis, these associations have not been established yet for the oral microbiome. Because the study of microbiota is still in its infancy the significance of these associations and possible therapeutic implications are not clear now. Studies are small and there is a lack of standardization in the landscape of microbiome techniques. There is a sense of urgency to improve reproducibility between laboratories. Nevertheless, the future of microbiota research seems bright. The role of the oral microbiome has to be elucidated further.

\section{References}

1. Murphy KP (2011) Janeway's immunobiology: $8^{\text {th }}$ edn., New York:Garland Science,Taylor\&Francis Group, London, pp.465-66.

2. Van Wijk F, Cheroutie H (2009) Intestinal T cells: facing the mucosal immune dilemma with synergy and diversity. Seminars in Immunology 21(3): 130-138.

3. Azuma M (2006) Fundamental mechanisms of host immune responses to infection. Journal of Periodontol Research 41(5): 361-373.

4. Radtke AL, O Riordan MX (2006) Intracellular innate resistance to bacterial pathogens. Cell Microbiology 8(11): 1720-1729.

5. Janeway CA, Medzhitov R (2002) Innate immune recognition. Annual Review of Immunology 20: 197-216.

6. Tlaskolova Hagenova H, Tuckova L, Mestecky J, et al (2005) Scand.J.Immunol 62: 106-113.

7. Pancer Z, Cooper MD (2006) The evolution of adaptive immunity. Annual Review of Immunology 24: 497-518.

8. Kutta H, May J, Jaehne M, A Münscher, FP Paulsen (2006) Antimicrobial defense mechanism of the human parotid duct. Journal of Anatomy 208(5): 609-619.

9. Abiko Y, Nishimura M, Kaku T (2003) Defensins in saliva and the salivary glands. Medical Electron Microsccopy 36(4): 247-252.

10. Naafs Michael AB (2018) The Antimicrobial Peptides: Ready for Clinical Trials? Infection Control.tips: submitted for publication.

11. von Delwig A, Bailey E, Gibbs DM, Robinson JH (2002) The route of bacterial uptake by macrophages influences the repertoire of epitopes presented to CD4 cells. European Journal of Immunology 32(12): 37143719. 
12. Metzger Z (2000) Macrophages in periapical lesions. Endodontics and Dental Traumatology 16(1): 1-8.

13. Smith DJ, King WF, Akita H, Taubman MA (1998) Association of salivary immunoglobulin antibody and initial streptococcal mutans infection. Oral Microbiology and Immunology 13(5): 278-285.

14. Page RC (1998) The pathobiology of periodontal diseases may affect systemic diseases: inversion of a paradigm. Annals of Periodontology 3(1): 108-120.

15. Roitt IM, Delves PJ (2001) Roitt's Essential Immunology,Oxford Blackwell Sience, UK.

16. Springer TA (1990) Adhesion receptors of the immune system. Nature 346(6283): 425-434.

17. Renglli HH (1977) Phagocytosis and killing by crevicular neutrophils. In: Lehner T, Editor. The Borderland Between Caries and Periodontal Disease, London: Academic Press, UK, pp. 211-222.

18. Haas W, Pereira P, Tonegawa S (1993) Gamma/delta cells. Annual Review of Immunology 11: 637-685.

19. Germain RN (1994) MHC-dependent antigen processing and peptide presentation: providing ligands for T lymphocyte activation. Cell 76(2): 287-299.

20. Lenschow DL, Walumas TL, Bluestone JA (1996) CD 28/B 7 system of T cell co-stimulation. Annual Review of Immunology 14: 233-258.

21. Liu YJ (2005) IPC: professional type 1 interferon producing cells and plasmacytoid dendritic precursors. Annual Review of Immunology 23: 275-306.

22. Bharadway AS, Bewtra AK, Agrawal DK (2007) Dendritic cells in allergic airway inflammation. Canadian Journal of Physiology and Pharmacology 85(7): 688-699.

23. Maloy K, Powrie F (2001) Regulatory T cells in the control of immune pathology. Nature Immunology 2(9): 816-822.

24. Lechler RI, Sykes M, Thomson AW, Turka LA (2005) Organ transplantation-how much of the promise has been realized? Nature Medicine 11(6): 605-613.

25. Chong AS, Alegre ML (2012) The impact of infection and tissue damage in solid-organ transplantation. Nature Reviews Immunology 12(6): 459471.

26. Hlava N, Niemann CU, Gropper MA (2009) Postoperative infectious complications of abdominal solid organ transplantation. Journal of Intensive Care Medicine 24(1): 3-17.

27. Winters HA, Parbhoo RK, Scafer JJ, Goff DA (2011) Extended-spectrum beta lactamase-producing bacterial infections in adult solid organ transplant recipients. The Annals of Pharmacotherapy 45(3): 309-316.

28. Brown LR, Mackler BF, Levy BM, Wright TE, Handler SF et al. (1979) Comparison of the plaque microflora in immunodeficient and immunocompetent dental patients. Journal of Dental Research 58(12): 2344-2352.

29. Martirostan G, Radosz Komoniewska H, Pietrzak B, Ekiel A, Kamiński $P$ et al. (2012) Characterization of vaginal lactobacilli in women after kidney transplantation. Anaerobe 18(2): 209-213.

30. Diaz PI, Hong BY, Frias Lopez J, Amanda K Dupuy, Mark Angeloni, et al. (2013) Transplantation-Associated Long-Term Immunosuppression Promotes Oral Colonization by Potentially Opportunistic Pathogens without Impacting Other Members of the Salivary Bacteriome. Clinical and Vaccine Immunology 20(6): 920-930.

31. Kim YJ, Yoon JH, Kim SJ, Hong KW, Kim JI, et al. (2011) High mortality associated wiyh Acinetobacter species infections in liver transplant patients. Transplantation Proceedings 43(6): 2397-2399.
32. Lee SO, Kang SH, Abdel Massih RE, Brown RA, Razonable RR, et al. (2011) Spectrum of early-onset and late-onset bacteremias after liver transplantation: implications for management. Liver Transplantation 17(6): 733-741.

33. Chen B, Sun L, Zhang X (2017) Integration of microbiome and epigenome to decipher the pathogenesis of autoimmune diseass. Journal of Autoimmunity 83: 31-42.

34. Zhang X, Zhang D, Jia H, Feng Q, Wang D, et al. (2015) The oral and in rheumatoid arthritis and gut microbiomes are perturbed in rheumatoid arthritis and partly normalized after treatment. Nature Medicine 21(8): 895-905.

35. Vaghef Mebrabany E, Alipour B, Homayoini Rad A, Sakineh Khatoon Sharif, Mohammad Asghari Jafarabadi, et al. (2014) Probiotic supplementation improves inflammatory status in patients with rheumatoid arthritis. Nutrition 30(4): 430-435.

36. Zamani B, Golkar HR, Farshbub S, Emadi Baygi M, Tajabadi Ebrahimi M, et al. (2016) Clinical and metabolic response to probiotic supplementation in patients with rheumatoid arthritis. International Journal of Rheumatic Diseases 19(9): 869-879.

37. Szymala A, Rosenthal J, Szczerba BM, Harini Bagavant, Shu Man Fu, et al. (2014) T cell epitope mimicry between Sjögren's syndrome Antigen A(SSA) Ro 60 and oral gut skin and vaginal bacteria. Clinical Immunology 152(0): $1-9$

38. van der Meulen T, Kroese FG, Liefers SC, Arnau Vich Vila, Hermie JM, et al. (2017) Is the Oral Microbiome Involved in the Pathogenesis of Sjögren's Syndrome? ACR/ARHP Annual Meeting, Abstract nr: 569.

39. Correa JD, Calderaro DC, Fereira GA (2017) Subgingival microbiota dysbiosis in systemic lupus erythematosus: association with periodontal status. Microbiome 5(1): 34.

40. Volkmann ER, Hoffman Vold A, Chang YL (2017) Systemic sclerosis is associated with specific alterations in gatrointestinal microbiota. BMJ Open Gastroenterology 4(1): e00134.

41. Seoidi N, Bergmauer LA, Drobniewski F, Paster B, Fortune F (2015) The oral mucosa land salivary microbial community of Behcet's syndrome and recurrent aphthous stomatitis. Journal of Oral Microbiology 7 : 27150.

42. Yang L, Wang L, Wang X, Cory J Xian, Hai Lu (2016) A Possible Role of Intestinal Microbiota in the Pathogenesis of Ankylosing Spondylitis. International Journal of Molecular sciences 17(12): 2126.

43. Taurog JD, Richardson JA, Croft JT, Simmons WA, Zhou M, et al. (1994) The germfree state prevents development of gut and joint inflammatory disease in HLA-B 27 transgenic rats. The Journal of Experimental Medicine 180(6): 2359-2364

44. Rath HC, Herfurth HH, Ikeda JS, Grenther WB, Hamm TE, et al. (1996) Normal luminal bacteria especially Bacteroides species mediate chronic colitis,gastritis and arthritis in HLA-B27 human beta2 microgobulin transgenic rats. The Journal of Clinical Investigation 98(4): 945-953.

45. Bisanz JE, Suppiah P, Thomson WM, Trudy Milne, Nigel Yeoh, et al. (2016) The oral microbiome of patients with axial spondyloarthritis compared to healthy individuals. Peer.J: 2095.

46. Ogrendik M (2015) Periodontal pathogens are likely to be responsible for the development of ankylosing spondylitis. Current Rheumatology Reviews 11(1):47-49.

47. Sparks JA, Costenbader KK (2014) Genetics, environment and geneenvironment interactions of systemic rheumatic diseases. Rheumatic Diseases Clinics of North America 40(4): 637-657.

48. Rosenbaum JT, Lin P, Asquith M (2016) The microbiome, HLA and the pathogenesis of uveitis. Japanese Journal of Ophthalmology 60(1): 1-6. 
49. Martinez A, Pacheco Tena C, Vaquez Mellado J (2004) Relationship between disease activity and infection in patients with spondylarthropathies. Annals of the Rheumatic Diseases 63(10): 1333813340 .

50. Costello ME, Ciccia F, Willner D, Warrington N, Robinson PC, et al. (2015) Intestinal dysbiosis in ankylosing spondylitis. Arthritis \& Rheumatology 67(3): 686-691.

51. Rodriquez IK, Andrigueti M, de Oliviera GI, de Lucca Schiavon L, de Andrade KR, et al. (2015) An investigation into the relationship between anti-Helicobacter pylori and anti-Saccharomyces cerevisae antibodies in patients with axial spondylarthritis and Crohn disease. Rheumatology International 35(2): 359-366.

52. Ciccia F, Rizzo A, Triolo G (2016) Subclinical gut inflammation in ankylosing spondylitis. Current Opinion in Rheumatology 28(1): 89-96.

53. Costello ME, Elewaut D, Kenna TJ, Brown MA (2013) Microbes,the gut and ankylosing spondylitis. Arthritis Research \&Therapy 15(3): 214.

54. Rosenbaum JT, Davey MP (2011) Time for a gut check:Evidence for the hypothesis that HLA-B 27 predisposes to ankylosing spondylitis by altering the microbiome. Arthritis and Rheumatism 63(11): 3195-3198.

55. Tito RY, Cypers H, Joossens M, Varkas G, Van Praet L, et al. (2016) Dialister as a microbial marker of disease activity in spondyloarthritis. Arthritis \& Rheumatology 69(1): 114-121.

56. Talotta R, Alzeni F, Ditto MC, Maria Chiara Gerardi, Piercarlo Sarzi Puttini (2017) The Microbiome in Connective Tissue Disorders and Vasculitides: An Updated Narrative Review. Journal of immunology Research 63(36): 498.

57. Clifford A, Hoffman GS (2015) Evidence for a vascular microbiome and its role in vessel health and disease. Current Opinion in Rheumatology 27(4): 397-405.

58. Bhatt AS, Manzo VE, Pedamulla CS, Duke F, Cai D, et al. (2014) In search of a candidate pathogen for giant cell arteriitis: sequencing based on characterization of the giant cell microbiome. Arthritis \& Rheumatology 66(7): 1339-1344.

59. Kinumaki A, Sekizuk A, Hamada H, Kengo Kato, Akifumi Yamashita, et al. (2015) Characterization of the gut microbiota of Kawasaki disease patients by metagnomic analysis. Frontiers in Microbiology 6: 824 .

60. Yung YS, Shin MH, Kweon SS, Young Hoon Lee, Ok Joon Kim, et al. (2015) Periodontal disease associated with blood glucose levels in urban Korean aged 50 years and older: The Dong -gu study. Gerodontology 32(4): 267-273.

61. Morita I, Inagaki K, Nakamura F, Noguchi T, Matsubara T, et al. (2012) Relationship between perodontal status and levels of glycated hemoglobin. Journal of Dental Research 91(2): 161-166.

62. Bharti P, Katagiri S, Nitta H, Nagasawa T, Kobayashi H, et al. (2013) Periodontal treatment with topical antibiotics improves glycemic control in association with elevated serum adinopectin in patients with type2 diabetes mellitus. Obesity Research \& Clinical Practice 7(2): e129-e138.

63. Moeintaghavi A, Arab HR, Bozorgnia Y, Kianoush K, Alizadeh M, et al. Non-surgical periodontal therapy affects metabolic control in diabetes: a randomized controlled clinical trial. Australian Dental Journal 57(1): 31-37.

64. Chee B, Park B, Bartold PM (2013) Periodontitis and type2 diabetes: a two-way relationship. International Journal of Evidence Based Healthcare 11(4): 317-329.

65. PM Preshaw, AL Alba, D Herrera, S Jepsen, A Konstantinidis, et al. (2012) Periodontitis and diabetes: a two-way relationship.Diabetologica 55(1): 21-31.

66. Levine RS (2013) Obesity, diabetes and periodontitis, a triangular relationship? British Dental Journal 215(1) 33-39.
67. Makiura N, Ojima M, Koy M, Furuta N, Okahashi N, et al. (2008) Relationship of Porphyromones gingivalis with glycemic level in patients with type 2 diabetes following periodontal treatment. Oral Microbiology and Immunology 23(4): 348-351.

68. Aamaimanan P, Amimonan P, Taweechaisupapong S (2013) Quantification of key periodontal pathogens in insulin-dependent type2 diabetes and non-diabetic patients with generalized periodontitis. Anaerobe 22: 64-68.

69. Hyvarinen K, Salminen A, Salomea V, Pussinen PJ (2015) Systemic expsure to a common periodontal pathogen and missing teeth are associated with metabolic syndrome Acta Diabetologica 52(1): 179-182.

70. Thorstensson H, Dalden G, Hogosson A (1995) Some suspected periodontol pathogens and serum antibody response in adult longduration insulin-dependent diabetics. Journal of Clinical Periodontology 22(6): 449-458.

71. Shilittoe E, Weistock R, Kim T, Simon H, Planer J, et al. (2012) The oral microflora in obesity and type 2 diabetes. Journal of Oral Microbiology 4: 19013

72. Kampoo K, Teanpaisan R, Ledder RG, Andrew J McBain (2014) Oral bacterial communities in individuals with type2 diabetes who live in Thailand. Applied and Environmental Microbiology 80(2): 662-671.

73. Long J, Cai Q, Steinwandel M, Hargreaves MK, Bordenstein SR, et al. (2017) Association of oral microbiome with type 2 diabetes risk. Journal of Periodontal Research 52(3): 636-643.

74. Algert CS, McEldulff A, Morris JM, Roberts CL (2009) Prenatal risk factors for early onset type 1 diabetes in a 2000-2005 birth cohort. Diabetic Medicine 26(12): 1193-1197.

75. Alexandra E, Livanos TU, Vangay P, Pathmasiri W, Stewart D, et al. (2016) Antibiotic-mediated gut microbiome perturbation accelerates development of type 1 diabetes in mice. Nature Microbiology 1(11): 16140.

76. Naafs MAB (2018) Microbial Endocrinology in Microbiology: A MiniReview. International Journal of Clinical Endocrinology 2(1): 004-010.

77. Kodukula K, Faller DV, Harp DN, Iphigenia Kanara, Julie Pernokas, et al. (2017) Gut Microbiota and Salivary Diagnostics: The Mouth Is Salivating to Tell Us Something. BioRes Open Access 6(1): 123-132.

78. Lerner A, Jeremia P, Matthias T (2017) Gut-thyroid axis and celiac disease. Endocrine Connections 6(4): R52-R58.

79. Lerner A, Aminov R, Matthias $T$ (2016) Dysbiosis may trigger autoimmune diseases via inappropriate post-translational modification of host proteins. Frontiers in Microbiology 7: 84.

80. Lerner A, Matthias $\mathrm{T}$ (2015) Changes in intestinal tight junction permeability associated with industrial food additives explain the rising incidence of autoimmune disease. Autoimmunity Reviews 14(6): 479489.

81. Kyriacon K, McLaughlin J, Akheel A (2015) Thyroid disorders and gastrointestinal and liver dysfunction: a state of the art review. European Journal of Internal Medicine 26(8): 563-571.

82. Mori K, Nakagawa Y, Ozaki H (2012) Does the gut microbiota trigger autoimmune thyroiditis? Discovery Medicine 14(78): 321-326.

83. Duntas LH (2009) Does celiac disease trigger autoimmune thyroiditis? Nature Reviews Endocrinology 5(4): 190-191.

84. Lerner A, Matthias T (2016) GUT-the troyan horse in remot's organ autoimmunity. Journal of Clinical \& Cell Immunology 7: 401.

85. Lerner A, Matthias T (2016) Nutrients,bugs and us: the short-chain fatty acids story in celiac disease. International Journal of Celiac Disease $4(3)$ : 92-94. 
86. Lerner A, Aminov R, Matthias $T$ (2017) Intestinal dysbiotic transglutaminases are potential environmental drivers of systemic autoimmunogenesis. Frontiers in Microbiology 8: 66.

87. Heron SE, Elahi S (2017) HIV Infection and Compromised Mucosal Immunity: Oral Manifestations and Systemic Inflammation. Frontiers in Immunology 8: 241.

88. Elahi S, Weiss RH, Merani S (2016) Atorvastine restricts HIV replication in CD4+ T cells by upregulation of p21. AIDS 30(2): 171-183.

89. Leng SX, Margolick JB (2015) Understanding frailty aging and inflammation in HIV infection. Current HIV/AIDS Reports 12(1): 25-32.

90. Sankaran S, Guadelupe M, Reay E, George MD, Flamm J, et al. (2005) Gut mucosal $\mathrm{T}$ cell responses and gene expression correlate with protection against disease in long-term HIV-1-infected nonprogressors. Proceedings of the National Academy of Sciences of the United States of America 102(28): 9860-9865.

91. Nilsson J, Kinloch de Loes S, Granath A, Sönnerborg A, Goh LE, et al. (2007) Early immune activation in gut-associated and peripheral lymphoid tissue during acute HIV infection. AIDS 21(5): 565-574.

92. Kim CJ, Mc Kumon LR, KOvacs CM, Kandel G, Huibner S, et al. (2013) Mucosal Th17 cell function is altered during HIV infection and is an independent predictor of systemic immune activation. Journal of Immunology 19(5): 2164-2173.

93. Klatt NR, Chomont N, Donek DC, Deeks SG (2013) Immune activation and HIV persistence: implications for curative approaches to HIV infection. Immunological Reviews 254(1): 362-342.

94. Brenchley JM, Donek DC (2008) The mucosal barrier and immune activation in HIV pathogenesis. Current Opinion in HIV and AIDS 3(3): 356-361.

95. Wu RQ, Zhang ZF, Tu E, Chen QM, Chen W (2014) The mucosal immune system in the oral cavity: an orchestra of T cell diversity. International Journal of Oral Science 6(3): 125-132.

96. Mc Clure R, Massari P (2014) TLR-dependent human mucosal epithelial cell responses to microbial pathogens. Frontiers in Immunology 5: 386.

97. Eke PI, Dye BA, Wei L Periodontal Disease Surveillance Workgroup.

98. Beck J, Eke PI, Dye BA, Wei L, Slade GD, et al. (2012) Prevalence of periodontitis in adults in the United States 2009 and 2010. Journal of Dental Research 91(10): 914-920.

99. Li Y, Saxena D, Chen Z, Liu G, Abrams WR, et al. (2014) HIV infection and microbial diversity in saliva. Journal of Clinical Microbiology 52(5): 1400-1411.

100. Malamud D, Wald SM (2010) The mouth: a gateway or a trap for HIV? AIDS 24(1): 5-16.

101. Vaseliu N, Kamiru HN, Kabue M (2010) Oral manifestations of HIV infection. Contemp.Clin.Dent. 1(1): 5-10.

102. Challecombe SJ, Naglik JR (2006) The effects of HIV infection on oral mucosal immunity. Advances in Dental Research 19(1): 29-33.

103. Nittayananta W, Mitarnan W, Talungshit S, Hutcha Sriplung (2012) Changes in oral cytokeratin expression in HIV-infected subjects with long-term use of HAART. Oral Diseases 18(8): 793-801.

104. Dang AT, Cotton S, Sankaran Walter S (2012) Evidence of an increased pathogenic footprint in the lingual microbiome of untreated HIV infected patients. BMC Microbiology 12: 153.

105. Mukherjee PK, Chandra J, Retuerto M, Masoumeh Sikaroodi, Robert E Brown, et al. (2014) Oral microbiome analysis of HIV-infected patients:identification of Pichia as antagonnist of opportunistic fungi. Plos Pathogen 10(3): e 1003996.
106. Kistler JO, Arirachakaran P, Poovorawan Y, Dahlén G, Wade WG, et al. (2015) The oral microbiome in human immunodeficiency virus (HIV)positive individuals. Journal of Medical Microbiology 64(9): 10941101.

107. Moyes DL, Saxena D, John MD, Malamud D (2016) The gut and oral microbiome in HIV disease: a workshop report. Oral Diseases 22(Suppl.1): 166-170

108. Costa PI, Zeller G, Sunagawa S, Pelletier E, Alberti A, et al. (2017) Towards standards for human fecal sample processing in metagnomic studies. Nature Biotechnology 35(11): 1069-1076.

109. Tighe S, Afshinnekoo E, Rock TM (2017) Genomic Methods and Microbiological Technologies for Profiling Novel and Extreme Environments for the Extreme Microbiome Project (XMP). Journal of Biomolecular Techniques 28(1): 31-39.

110. McCoy KD, Geuking MB, Rouchi F (2017) Gut Microbiome Standardization in Control and Experimental Mice. Current Protocols in Immunology 117(23): 1-23.

111. EpsteinSE,ZhouYE,ZhuJ(1999)Infectionand atherosclerosis:emerging mechanistic paradigms. Circulation 100(4): e20-e28.

112. Petersen PE, Ogawa H (2012) The global burden of periodontal disease: towards integration with chronic disease prevention and control. Periodontology 60(1): 15-39.

113. Coogan MM, Greenspan J, Chollacombe SJ (2005) Oral lesions in infection with human immunodeficiency virus. Bulletin of the World Health Organization 83(9): 700-706.

114. Palmer GD, Robinson PG, Challacombe SJ, Birnbaum W, Croser D, et al. (1996) Aetiological factors for oral manifestations of HIV infection. Oral Diseases 2(3): 193-197.

115. Moutsopoulos NM, Madianos PN (2006) Low grade inflammation in chronic infectious diseases: paradigm of periodontal infections. Annals of the NewYork Academy of Sciences 1088: 252-264.

116. Li X, Kollveit KM, Tronstad L, Olsen I (2000) Systemic diseases caused by oral infection. Clinical Microbiology Reviews 13(4): 547-558.

117. Haraszthy VI, Zambon JJ, Trevison M, Zeid M, Genco RJ (2000) Identification of periodontal pathogens in atheromatous plaques. Journal of Periodontology 71(10): 1554-1560.

118. Steinman RM (2003) Some interfaces of dendritic cell biology. APMIS 111(7-8): 675-697.

119. Steinman RM (2007) Dendritic cells: understanding immunogenicity. European Journal of Immunology 37(Suppl.1): S53-S60.

120. Jotwani R, Palucka AK, Al Quatab M, Mahyar Nouri Shirazi, Jay Kim, et al. (2001) Mature dendritic cells infiltrate the T cell-rich region of oral mucosa in chronic periodontitis: in situ, in vivo and in vitro studies. Journal of Immunology 167(8): 4693-4700.

121. Wray D, Rees SR, Gibson J (2000) The role of allergy in oral mucosal diseases. QJM 93(8): 507-511.

122. Cassani B, Villablanca EJ, Quintana FJ, Love PE, Lacy Hulbert A, et al. (2011) Gut-tropic T cells that express integrin alpha4beta7 and CCR9 are required for induction of oral immune tolerance in mice. Gastroenterology 14(6): 2109-2118.

123. Wang J, Toes RE (2008) Mechanisms of oral tolerance revisited. Arthritis Research \& Therapy 10(2): 108.

124. Weiner HL, da Cunho AP, Quintana F, Wu H (2011) Oral tolerance. Immunological Reviews 24(1): 241-259.

125. Resigno M (2011) Dendritic cells in oral tolerance in the gut. Cellular Microbiology 13(9): 1312-1318. 
126. Coombes JL, Powrie F (2008) Dendritic cells in intestinal immune regulation. Nature Reviews. Immunology 8(6): 435-446.

127. Kosten IJ, van de Ven R, Thon M (2017) Comparative phenotypic and functional analysis of migratory cell subsets from human oral mucosa and skin. Plos One 12(7): e1800333.

128. Naafs Michael AB (2018) The Burden of Allergic Rhinitis: A MiniReview. Global Journal of Otolaryngology 13(1): 555854.

129. Kamada N, Seo SU, Chen GY, Núñez G (2013) Role of the gut microbiota in immunity and inflammatory disease. Nature Reviews.Immunology 13(5): 321-335.

130. Hill DA, Artis D (2010) Intestinal bacteria and the regulation of immune cell homeostasis. Annual Review of Immunology 28: 623-667.

131. Hooper LV, Littman DR, McPherson AJ (2012) Interactions between the microbiota and the immune system. Science 336(6086): 1268-1273.
132. Ostman S, Rask C, Wold AE, Hultkrantz S, Telemo E (2006) Impaired regulatory $\mathrm{T}$ cell function in germ-free mice. European Journal of Immunology 36(9): 2336-2346.

133. Tanabe S (2013) The effect of probiotics and gut microbiota on Th17 cells. International Reviews of Immunology 32(5-6): 511-525.

134. Pontahidis T, Kearney SM, Levkovich T, Peimin Qi, Bernard J Varian, et al. (2013) Microbial symbionts accelerate wound healing via the neuropeptide hormone oxytocin. Plos One 8: e78898.

135. Reiche EM (2004) Stress, depression, the immune system and cancer. The Lancet Oncology 5(10): 617-625.

136. Lyte M (2004) Microbial endocrinology and infectious disease in the 21th century. Trends in Microbiology 12(1): 14-20.

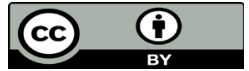

This work is licensed under Creative Commons Attribution 4.0 License

To Submit Your Article Click Here:

Submit Article

DOI: 10.32474/MADOHC.2018.01.000109

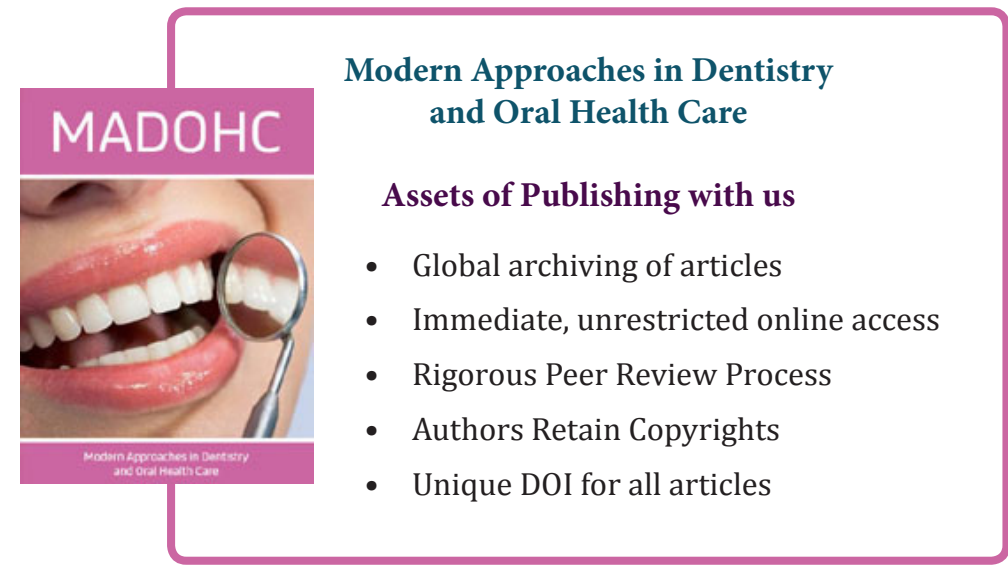

